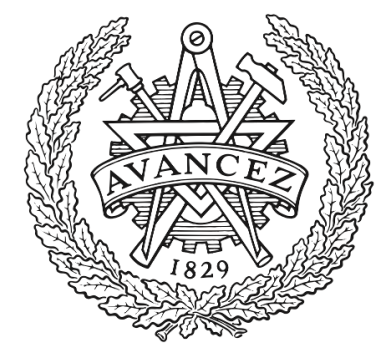

CHALMERS

UNIVERSITY OF TECHNOLOGY

\title{
Combustion-induced local shear layers within premixed flamelets in weakly turbulent flows
}

Downloaded from: https://research.chalmers.se, 2023-04-26 12:11 UTC

Citation for the original published paper (version of record):

Lipatnikov, A., Sabelnikov, v., Nishiki, S. et al (2018). Combustion-induced local shear layers within premixed flamelets in weakly turbulent flows. Physics of Fluids, 30(8): 1-10.

http://dx.doi.org/10.1063/1.5040967

N.B. When citing this work, cite the original published paper. 


\title{
Combustion-induced local shear layers within premixed flamelets in weakly turbulent flows
}

\author{
A. N. Lipatnikov, ${ }^{1, a)}$ V. A. Sabelnikov, ${ }^{2,3}$ S. Nishiki, ${ }^{4}$ and T. Hasegawa ${ }^{5}$ \\ ${ }^{1}$ Department of Mechanics and Maritime Sciences, Chalmers University of Technology, \\ Gothenburg 412 96, Sweden \\ ${ }^{2}$ ONERA-The French Aerospace Laboratory, F-91761 Palaiseau, France \\ ${ }^{3}$ Central Aerohydrodynamic Institute (TsAGI), 140180 Zhukovsky, Moscow Region, Russian Federation \\ ${ }^{4}$ Department of Mechanical Engineering, Kagoshima University, Kagoshima 890-0065, Japan \\ ${ }^{5}$ Institute of Materials and Systems for Sustainability, Nagoya University, Nagoya 464-8603, Japan
}

(Received 22 May 2018; accepted 16 July 2018; published online 6 August 2018)

\begin{abstract}
3D direct numerical simulation data obtained from statistically stationary, planar, weakly turbulent, premixed flames, which are characterized by two different density ratios (7.53 and 2.50) and are associated with the flamelet combustion regime, are analyzed to investigate differences between velocity and pressure variations (i) in flamelets in a weakly turbulent flow and (ii) in the counterpart laminar flame. Results show that while the thermo-chemical structure of the flamelets is weakly affected by turbulence under the studied conditions, the local velocity, vorticity, and pressure fields within the flamelets differ significantly from the velocity, vorticity, and pressure fields, respectively, within the laminar flame. In particular, local shear layers appear within flamelets in the turbulent flow because acceleration of a reacting mixture by the local pressure gradient is inversely proportional to the mixture density and, hence, depends on the mixture state. The shear layers are characterized by large velocity gradients (both the tangential gradient of the normal velocity with respect to the flamelet surface and the normal gradient of the tangential velocity), whose magnitudes may be comparable with the magnitude of the velocity gradient across the laminar flame. In flamelet zones characterized by a relatively large magnitude of the locally normal gradient of the tangential velocity, the local vorticity magnitude is also large and such zones contribute substantially to the overall generation of vorticity due to baroclinic torque. These results cast doubts on the validity of a simple common modeling approach that consists in directly invoking expressions derived for the laminar flames in order to describe the influence of combustion-induced thermal expansion on weakly turbulent velocity and pressure fields. Published by AIP Publishing. https://doi.org/10.1063/1.5040967
\end{abstract}

\section{INTRODUCTION}

When a premixed flame propagates in a turbulent medium, (i) the turbulence affects the flame and can significantly accelerate its propagation, while (ii) pressure perturbations due to combustion-induced thermal expansion affect the turbulent flow also. Although these two types of effects are closely linked with one another, e.g., combustion-induced changes of turbulence characteristics are widely assumed to substantially change the flame speed, the vast majority of available models of premixed turbulent burning place the focus of consideration on one of them. Pioneering models of the influence of turbulence on a premixed flame were developed by Damköhler ${ }^{1}$ and Schelkin ${ }^{2}$ almost 80 years ago and significant progress made in studying such effects is reviewed elsewhere. ${ }^{3-6}$ About ten years after the seminal work by Damköhler ${ }^{1}$ and Schelkin, ${ }^{2}$ Karlovitz et al. ${ }^{7}$ and, subsequently, Scurlock and $\mathrm{Grover}^{8}$ attracted attention of the combustion community to the influence of thermal expansion on turbulence in flames. Since that, the problem of the so-called "flamegenerated turbulence," posed in those pioneering studies, was

a)Electronic mail: lipatn@chalmers.se. examined by various research groups, as reviewed elsewhere. ${ }^{9,10}$ However, developments in this area were rather moderate and have not yet resulted in elaborating a wellrecognized comprehensive approach with well-documented predictive capabilities.

The present paper aims at revealing one of the eventual causes of such a moderate progress in that area. The point is that almost all models of the influence of premixed combustion on turbulence, reviewed elsewhere, ${ }^{9,10}$ see also a paper by Tian and Lindstedt ${ }^{11}$ as a recent example, rely somehow on theoretical expressions that describe velocity and pressure variations in the laminar premixed flames. Such an approach is widely accepted, in particular, due to the lack of an alternative, sufficiently well developed theory of the influence of combustion on turbulence. However, as will be shown later, the approach is oversimplified because the local velocity and pressure variations within flamelets (i.e., within thin inherently laminar zones that separate fresh reactants and equilibrium combustion products in a turbulent flow and retain the structure of a perturbed laminar flame ${ }^{12-14}$ ) can significantly differ from local velocity and pressure variations within the purely laminar premixed flame even if the turbulence weakly perturb the thermo-chemical structure of the flamelet. 
The specific goal of the present paper is to show such differences by analyzing Direct Numerical Simulation (DNS) data computed by Nishiki et al. ${ }^{15,16}$ about 15 years ago.

The choice of this database which may appear to be outdated when compared to recent DNS data ${ }^{17-23}$ generated in the case of complex combustion chemistry and a high ratio of the rms turbulent velocity $u^{\prime}$ to the laminar flame speed $S_{L}$ requires comments. Since the focus of the following discussion is placed on the influence of combustion-induced thermal expansion on the velocity and pressure fields, a detailed description of complex combustion chemistry appears to be of secondary importance when compared to two other major requirements. First, in order to make the studied thermal expansion effects as strong as possible, heat release and density drop should be localized to sufficiently thin zones and the velocity jump across such zones should be sufficiently large when compared to the rms turbulent velocity $u^{\prime}$. In other words, the flamelet regime $^{12-14}$ of premixed turbulent combustion associated with a low $u^{\prime} / S_{L}$ should be addressed. The selected DNS data were indeed obtained for this regime, as discussed in detail elsewhere, ${ }^{24}$ whereas the vast majority of recent very advanced DNS studies attacked other combustion regimes characterized by a large $u^{\prime} / S_{L}$. Second, to better explore the thermal expansion effects, data obtained at significantly different density ratios $\sigma=\rho_{u} / \rho_{b}$ are required. The selected DNS database does satisfy this requirement because cases of $\sigma=2.5$ and 7.53 were simulated, with all other things being roughly equal. Therefore, the selected DNS data appear to be fully adequate to the major goal of the present study. Here, subscripts $u$ and $b$ designate unburned reactants and burned products, respectively.

The paper is organized as follows. In Sec. II, the DNS attributes are reported. Simulated results are discussed in Sec. III, followed by conclusions.

\section{DNS ATTRIBUTES}

Since the DNS data were discussed in detail elsewhere ${ }^{15,16}$ and were already used by various research groups, ${ }^{24-38}$ we will restrict ourselves to a brief summary of those compressible 3D simulations. They dealt with statistically planar, equidiffusive, adiabatic flames modeled by unsteady 3D continuity, Navier-Stokes, and energy equations, supplemented with a transport equation for the mass fraction $Y$ of a deficient reactant and the ideal gas state equation. Temperature-dependence of molecular transport coefficients was taken into account, e.g., $v=v_{u}\left(T / T_{u}\right)^{0.7}$. Combustion chemistry was reduced to a single reaction. The Lewis $L e$ and Prandtl Pr numbers were equal to 1.0 and 0.7 , respectively. Accordingly, the mixture state was characterized with a single combustion progress variable $c=$ $\left(T-T_{u}\right) /\left(T_{b}-T_{u}\right)=1-Y / Y_{u}$.

The computational domain was a rectangular box $\Lambda_{x} \times \Lambda_{y} \times \Lambda_{z}$, where $\Lambda_{x}=8 \mathrm{~mm}$ and $\Lambda_{y}=\Lambda_{z}=4 \mathrm{~mm}$. It was resolved using a uniform rectangular $(2 \Delta x=\Delta y=\Delta z$ ) mesh of $512 \times 128 \times 128$ points. The flow was periodic in $y$ and $z$ directions.

Using an energy spectrum $E(\kappa)$ proposed by Kraichnan, ${ }^{39}$ homogeneous isotropic turbulence was generated ${ }^{15}$ in a separate box and was injected into the computational domain through the left boundary $x=0$. The generated turbulence was characterized ${ }^{15}$ by $u_{0}^{\prime}=0.53 \mathrm{~m} / \mathrm{s}$ and an integral length scale

$$
L=\frac{3 \pi}{4} \frac{\int_{0}^{\infty} \kappa^{-1} E(\kappa) d \kappa}{\int_{0}^{\infty} E(\kappa) d \kappa}
$$

equal to $3.45 \mathrm{~mm}$. Accordingly, the turbulent Reynolds number $R e_{t}=u^{\prime} L / v_{u}=96$.

At $t=0$, a planar laminar flame was embedded into statistically the same turbulence assigned for the velocity field in the entire computational domain. Subsequently, the mean inflow velocity $U$ was increased twice, i.e., $U\left(0 \leq t<t_{I}\right)=$ $S_{L}<U\left(t_{I} \leq t<t_{I I}\right)<U\left(t_{I I} \leq t\right)=U_{T}\left(t_{I I}\right)$, in order to keep the flame in the computational domain until the end $t_{I I I}$ of the simulations. Here,

$$
U_{T}(t)=\frac{1}{\rho_{u} Y_{u} \Lambda_{y} \Lambda_{z}} \int_{0}^{\Lambda_{x}} \int_{0}^{\Lambda_{y}} \int_{0}^{\Lambda_{z}} W(x, y, z, t) d z d y d x
$$

is the turbulent burning velocity and $W$ is the mass rate of consumption of the deficient reactant.

Three DNS data sets $\mathrm{H}, \mathrm{M}$, and $\mathrm{L}$ associated with high, medium, and low, respectively, density ratios were originally generated by Nishiki et al. ${ }^{15,16}$ Since the focus of the present study is placed on thermal expansion effects, the following discussion will be restricted to comparison of the results obtained in two cases characterized by the highest and the lowest density ratios, i.e., flame $\mathrm{H}\left[\sigma=7.53, S_{L}=0.6 \mathrm{~m} / \mathrm{s}, \delta_{L}=0.217\right.$ $\left.\mathrm{mm}, \overline{U_{T}\left(t_{I I} \leq t \leq t_{I I I}\right)}=1.15 \mathrm{~m} / \mathrm{s}\right]$ and flame $\mathrm{L}[\sigma=2.5$, $S_{L}=0.416 \mathrm{~m} / \mathrm{s}, \delta_{L}=0.158 \mathrm{~mm}, \overline{U_{T}\left(t_{I I} \leq t \leq t_{I I I}\right)}=0.79$ $\mathrm{m} / \mathrm{s}]$. In both cases, $\overline{U_{T}\left(t_{I I} \leq t \leq t_{I I I}\right)} / S_{L}=1.9$. Here, $\delta_{L}=\left(T_{b}-T_{u}\right) / \max \{|\nabla T|\}$ is the laminar flame thickness. The two flames are well associated with the flamelet combustion regime, e.g., various Bray-Moss-Libby (BML) expressions hold in cases $\mathrm{H}$ and L; see Figs. 1-4 in a recent paper by Lipatnikov et al. ${ }^{24}$ Since turbulence decays along the direction $x$ of the mean flow, the turbulence characteristics are slightly different at the leading edges of the $\mathrm{H}$ - and L-flame brushes, e.g., $u^{\prime}=0.33 \mathrm{~m} / \mathrm{s}, \lambda=0.43 \mathrm{~mm}, \eta=0.075 \mathrm{~mm}, K a=0.06$, and $D a=$ 17.5 in case $\mathrm{H}$ and $u^{\prime}=0.38 \mathrm{~m} / \mathrm{s}, \lambda=0.47 \mathrm{~mm}, \eta=0.084 \mathrm{~mm}$, $K a=0.10$, and $D a=10.0$ in case L. Here, $D a=\tau_{T} / \tau_{c}$ and $K a=$ $\tau_{c} u^{\prime} / \lambda$ are the Damköhler and Karlovitz numbers, respectively, $\tau_{T}=\bar{k}^{3 / 2} /\left(u^{\prime} \bar{\varepsilon}\right)$ and $\tau_{c}=v /\left(\operatorname{Pr} S_{L}^{2}\right)$ are turbulence and flame time scales, respectively, $\lambda=u^{\prime} \sqrt{15 v / \bar{\varepsilon}}$ and $\eta=\left(v^{3} / \bar{\varepsilon}\right)^{1 / 4}$ are the Taylor and Kolmogorov length scales, respectively, $k$ $=\left(u_{k} u_{k}-\bar{u}_{k} \bar{u}_{k}\right) / 2$ and $\varepsilon=2 v S_{i j} S_{i j}$ are the turbulent kinetic energy and its dissipation rate, respectively, the rms turbulent velocity $u^{\prime}$ is equal to $\sqrt{2 \bar{k} / 3}, S_{i j}=0.5\left(\partial u_{i} / \partial x_{j}+\partial u_{j} / \partial x_{i}\right)$ is the rate-of-strain tensor, $\bar{q}$ designates the mean value of a quantity $q$, and the summation convention applies for repeated indexes.

The DNS data were processed as follows. Mean quantities $\bar{q}(x)$ were averaged over a transverse plane $x=$ const. and over time (221 and 200 snapshots in cases $\mathrm{H}$ and $\mathrm{L}$, respectively, stored during a time interval of $t_{I I I}-t_{I I} \approx 1.5 L / u_{0}^{\prime} \approx 10 \mathrm{~ms}$ ). Subsequently, $x$-dependencies were mapped to $\bar{c}$-dependencies using the spatial profiles of the Reynolds-averaged combustion progress variable $\bar{c}(x)$. 
To generate a 3D joint Probability Density Function (PDF) $P\left[q_{1}, q_{2}, \bar{c}(x)\right]$ for quantities $q_{1}$ and $q_{2}$, intervals of $q_{1, \min } \leq q \leq q_{1, \max }$ and $q_{2, \min } \leq q \leq q_{2, \max }$ (e.g., $0 \leq c \leq 1$ if $q_{2}=c$ ) were divided into 100 bins each and sampling was performed in each point $\mathbf{x}$ for each snapshot. If one of the quantities is solely defined at $0<c<1$ (e.g., the components of the unit normal vector $\mathbf{n}=-\nabla c /|\nabla c|$ ), sampling was limited to points where $\epsilon<c(\mathbf{x}, t)<1-\epsilon$. Results reported in the following were obtained for $\epsilon=0.001$, with weak sensitivity of the results to variations in $\epsilon \ll 1$ being checked. Using the 3D joint PDF's, conventional PDF's $P\left(q_{1}\right)$ and values $\left\langle q_{1} \mid Q_{2}\right\rangle$ of the quantity $q_{1}$ conditioned to a value $Q_{2}$ of the quantity $q_{2}(\mathbf{x}, t)$, i.e., $Q_{2}-0.5 \Delta q_{2} \leq q_{2}(\mathbf{x}, t)<Q_{2}+0.5 \Delta q_{2}$, were evaluated as follows:

$$
\begin{gathered}
P\left(q_{1}\right)=\int_{q_{2, \min }}^{q_{2, \max }} P\left(q_{1}, \zeta, \bar{c}\right) d \zeta \\
\left\langle q_{1} \mid Q_{2}\right\rangle=\int_{q_{1, \min }}^{q_{1, \max }} \zeta P\left(\zeta, Q_{2}, \bar{c}\right) d \zeta\left[\int_{q_{1, \min }}^{q_{1, \max }} P\left(\zeta, Q_{2}, \bar{c}\right) d \zeta\right]^{-1}
\end{gathered}
$$

at various $\bar{c}(x)$ or $x$. Here, $\Delta q_{2}=\left(q_{2, \max }-q_{2, \min }\right) / 100$.

\section{RESULTS AND DISCUSSION}

\section{A. Velocity and pressure jumps at flamelets}

Shown in Fig. 1 are mean normalized velocity and pressure "jumps" at flamelets, evaluated as follows. First, a reaction surface $x_{f}=x_{i}\left(y_{j}, z_{k}, t_{n}\right)$ was determined using the following constraints: (i) $0.6<c\left(x_{i}, y_{j}, z_{k}, t_{n}\right)<0.9$, i.e., the reaction surface is inside the reaction zone characterized by substantial rate $W(\mathbf{x}, t)$ of product creation and (ii) $W\left(x_{i}, y_{j}, z_{k}, t_{n}\right)$ $>W\left(x_{i+1}, y_{j}, z_{k}, t_{n}\right)$, i.e., the reaction surface is associated with the local peak of $W(\mathbf{x}, t)$ along a ray $\left\{y=y_{j}, z=z_{k}\right\}$, which is normal to the mean flame brush. Second, for each grid point $\mathbf{x}_{f}=\left(x_{i}, y_{j}, z_{k}\right)$ that belongs to the reaction surface at instant $t_{n}$, the most close grid points $\mathbf{x}_{u}$ and $\mathbf{x}_{b}$ characterized by $c\left(\mathbf{x}_{u}, t_{n}\right)<\epsilon$ and $c\left(\mathbf{x}_{b}, t_{n}\right)>1-\epsilon$, respectively, were found. Third, the jump magnitudes $\Delta q=\left|q\left(\mathbf{x}_{u}, t_{n}\right)-q\left(\mathbf{x}_{b}, t_{n}\right)\right|$ were evaluated for various quantities $q$ such as (i) the velocity $u_{n}=$ $\mathbf{u} \cdot \mathbf{n}$ locally normal to the reaction surface, (ii) the magnitude $u_{t}=\left(\mathbf{u}_{t} \cdot \mathbf{u}_{t}\right)^{1 / 2}$ of the tangential velocity $\mathbf{u}_{t}=\mathbf{u}-u_{n} \mathbf{n}$, and (iii) the pressure $p$, with the unit vector $\mathbf{n}=\mathbf{n}\left(\mathbf{x}_{f}, t_{n}\right)$ being normal to the reaction surface. Fourth, the obtained values of $\Delta q\left(\mathbf{x}_{f}, t_{n}\right)$ were averaged over all grid points $\mathbf{x}_{f}$ on the reaction surface and over all instants $t_{n}$.

The solid line in Fig. 1(a) indicates that the mean jump $\overline{\left|u_{n, b}-u_{n, u}\right|}$ in the locally normal velocity agrees well with the velocity jump $\Delta u_{L}=(\sigma-1) S_{L}$ at the Unperturbed Laminar Flame Front (ULFF) in case H, thus, supporting the Simple Common Approach (SCA), i.e., the use of the results obtained from the laminar flames for modeling the influence of combustion-induced thermal expansion on a turbulent flow. The double-dashed-dotted line in the same figure shows substantial differences in $\overline{\left|u_{n, b}-u_{n, u}\right|}$ and $\Delta u_{L}$ at $\bar{c}<0.5$ in case $\mathrm{L}$. These differences appear to result from the relative weakness (due to a low $\sigma=2.5$ ) of the combustion-induced velocity perturbations when compared to the magnitude of turbulent velocity fluctuations. However, due to the relative weakness of the former perturbations in case $\mathrm{L}$, requirements to precision of modeling them could be reduced. Accordingly, the DNS data plotted as a double-dashed-dotted line in Fig. 1(a) do not seem to refute the SCA. Other curves shown in Fig. 1 will be discussed later.

The results reported in Fig. 2 do not seem to refute the SCA either. Indeed, broken curves indicate that, at various mean $\bar{c}$, normalized conditioned values $|\nabla p|_{L}^{-1}\left\langle T_{n} \mid c\right\rangle$ of the normal component $T_{n}=\mathbf{T} \cdot \mathbf{n}$ of the vector-term $\mathbf{T}$ on the Right-Hand

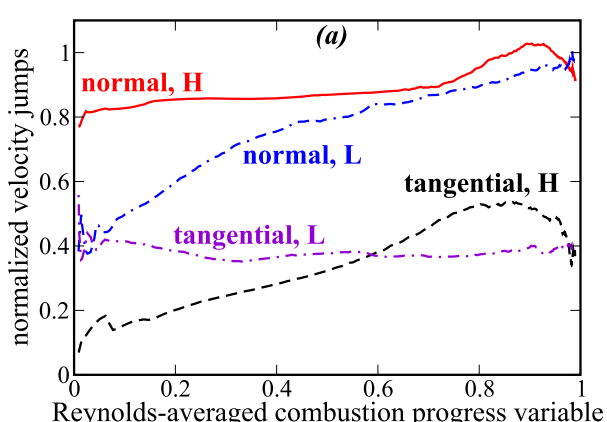

Reynolds-averaged combustion progress variable
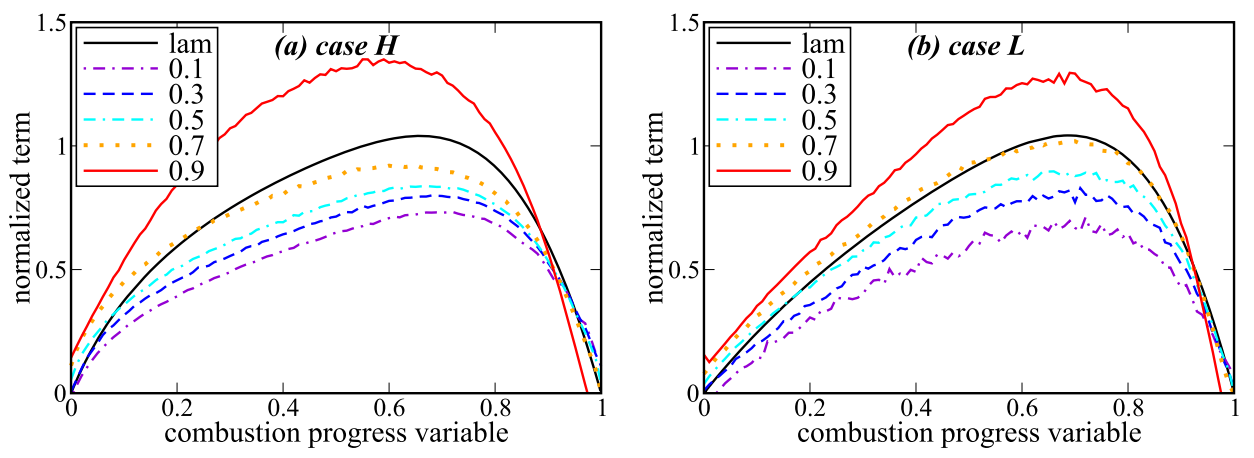

FIG. 1. Mean (a) velocity and (b) pressure jumps at flamelets, normalized using $\Delta u_{L}=(\sigma-1) S_{L}$ and $(\sigma-1) \rho_{u} S_{L}^{2}$, respectively.
FIG. 2. Normalized values $|\nabla p|_{L}^{-1}\left\langle T_{n} \mid c\right\rangle$ of the normal component $T_{n}=\mathbf{T} \cdot \mathbf{n}$ of the vector-term $\mathbf{T}$ on the right-hand side (RHS) of the Navier-Stokes equation, conditioned to the local value of $c(\mathbf{x}, t)$ and evaluated at various values of the Reynolds-averaged combustion progress variable $\bar{c}$ (broken lines), specified in legends, in cases (a) $\mathrm{H}$ and (b) L. Black solid lines show the results obtained from the laminar flames $\mathrm{H}$ and L. $|\nabla p|_{L}=(\sigma-1) \rho_{u} S_{L}^{2} / \delta_{L}$. 

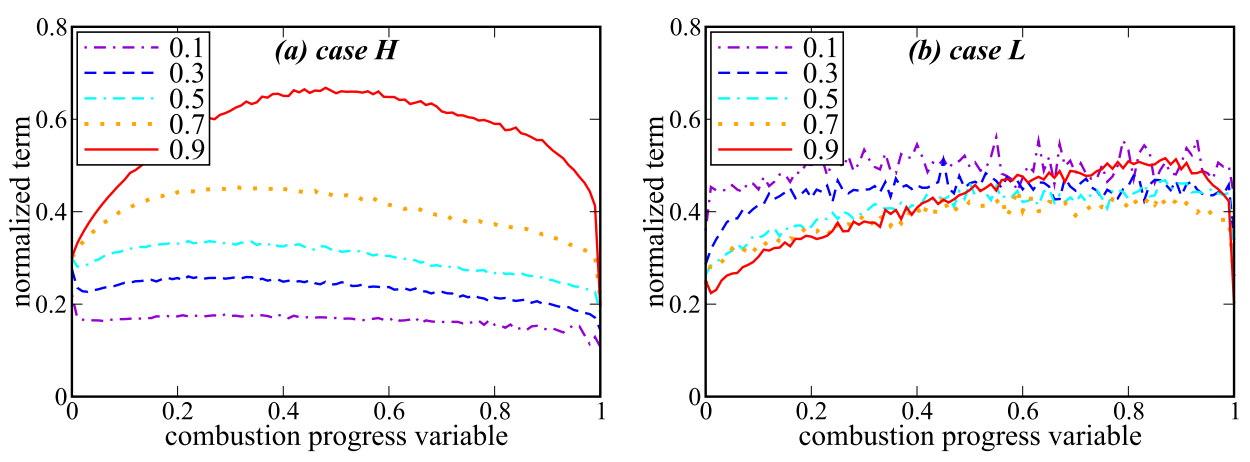

FIG. 3. Normalized values $|\nabla p|_{L}^{-1}\left\langle T_{t} \mid c\right\rangle$ of the magnitude $T_{t}$ of the tangential component $\mathbf{T}_{t}=\mathbf{T}$ $-T_{n} \mathbf{n}$ of the vector-term $\mathbf{T}$ on the RHS of the Navier-Stokes equation, conditioned to the local value of $c(\mathbf{x}, t)$ and evaluated at various values of the Reynolds-averaged combustion progress variable $\bar{c}$, specified in legends, in cases (a) $\mathrm{H}$ and (b) $\mathrm{L}$.
Side (RHS) of the Navier-Stokes equation

$$
\rho \frac{\partial u_{i}}{\partial t}+\rho u_{k} \frac{\partial u_{i}}{\partial x_{k}}=\underbrace{\frac{\partial \tau_{i, k}}{\partial x_{k}}-\frac{\partial p}{\partial x_{i}}}_{T_{i}}
$$

are sufficiently close to dependencies $T_{n}(c)$ computed in the ULFFs (black solid lines). Here, $q_{i}$ designates the $i$ th component of a vector $\mathbf{q}$,

$$
\tau_{i j}=\rho v\left(\frac{\partial u_{i}}{\partial x_{j}}+\frac{\partial u_{j}}{\partial x_{i}}-\frac{2}{3} \delta_{i j} \frac{\partial u_{k}}{\partial x_{k}}\right)
$$

is the viscous stress tensor, $\delta_{i j}$ is the Kronecker delta, and $|\nabla p|_{L}=(\sigma-1) \rho_{u} S_{L}^{2} / \delta_{L}$ is a characteristic magnitude of the pressure gradient in the ULFF.

While differences between $T_{n}(c)$ obtained from the laminar and turbulent flames are observed and are increased by $|\bar{c}-0.7|$, such effects are sufficiently weak when compared to the effects that will be discussed later. For instance, Fig. 3 shows that, contrary to the ULFF, the normalized conditioned tangential component $|\nabla p|_{L}^{-1}\left\langle T_{t} \mid c\right\rangle$ of the vector-term $\mathbf{T}$ does not vanish in the turbulent flames. While the magnitude of $\left\langle T_{t} \mid c\right\rangle$ is substantially less than the magnitude of $\left\langle T_{n} \mid c\right\rangle$, both magnitudes are of the same order. It is also worth noting that the results plotted in Figs. 3(a) and 3(b) are sufficiently close to one another in spite of the fact $|\nabla p|_{L}$ used to normalize these results is larger by a factor of about 6.6 in case $H$. Since the statistical characteristics of the turbulence are close in both cases, a comparison of Figs. 3(a) and 3(b) implies that $\left\langle T_{t} \mid c\right\rangle$ scales with $|\nabla p|_{L}$ to the leading order; i.e., the magnitude of $\left\langle T_{t} \mid c\right\rangle$ is significantly affected by the combustion-induced thermal expansion. Nevertheless, differences between curves plotted in Figs. 3(a) and 3(b) are also well pronounced, thus, indicating that the aforementioned scaling is approximate and effects of turbulence are not negligible.
Furthermore, Fig. 1(b) shows that the mean pressure jump $\overline{\left|p_{b}-p_{u}\right|}$ differs substantially from the pressure jump $\Delta p_{L}=(\sigma-1) \rho_{u} S_{L}^{2}$ at the ULFF in both cases $\mathrm{H}$ and L. Moreover, the dashed and dotted-dashed lines in Fig. 1(a) indicate a significant mean jump $\overline{\left|\mathbf{u}_{t, b}-\mathbf{u}_{t, u}\right|}$ in the locally tangential velocity, whereas the tangential velocity does not change at the ULFF.

Thus, the above results indicate that equations that describe local jumps in velocity, pressure, and vorticity at the ULFF do not hold in the case of turbulent burning even in the flamelet combustion regime characterized by $u^{\prime} \ll \sigma S_{L}$. Physical mechanisms responsible for the failure of the ULFF equations are explored in Sec. III B.

\section{B. Local shear layers and vorticity generation}

Figures 4 and 5 report the conditioned PDF's $P(c=0.5, \partial u / \partial y, \bar{c})$ and $P(c, \partial u / \partial y, \bar{c}=0.5)$, respectively, for a typical shear component $\partial u / \partial y$, with the results obtained for other shear components being qualitatively similar. The curves plotted in Figs. 4 and 5 are not smooth because selection of double-conditioned [to the local $c(\mathbf{x}, t)$ and to the mean $\bar{c}(x)]$ data significantly reduces the sampling ensemble. Nevertheless, the non-smooth shape of the curves does not seem to substantially affect the two qualitative trends emphasized in the following.

In particular, Figs. 4 and 5 show, first, that the conditioned PDF's $P(c=0.5, \partial u / \partial y, \bar{c})$ and $P(c, \partial u / \partial y, \bar{c}=0.5)$ are sufficiently wide at various mean $\bar{c}$ and various local $c(\mathbf{x}$, $t$ ). Second, the PDF half-widths are comparable in cases $\mathrm{H}$ (with the exception of $\bar{c}=0.9)$ and L, whereas the characteristic velocity gradient $|\nabla \cdot \mathbf{u}|_{L}=(\sigma-1) S_{L} / \delta_{L}$ within the ULFF, used to normalize the shear, is larger by a factor of about four in the former case. If the PDF's were solely controlled by turbulence, the H-half-widths would be smaller by approximately
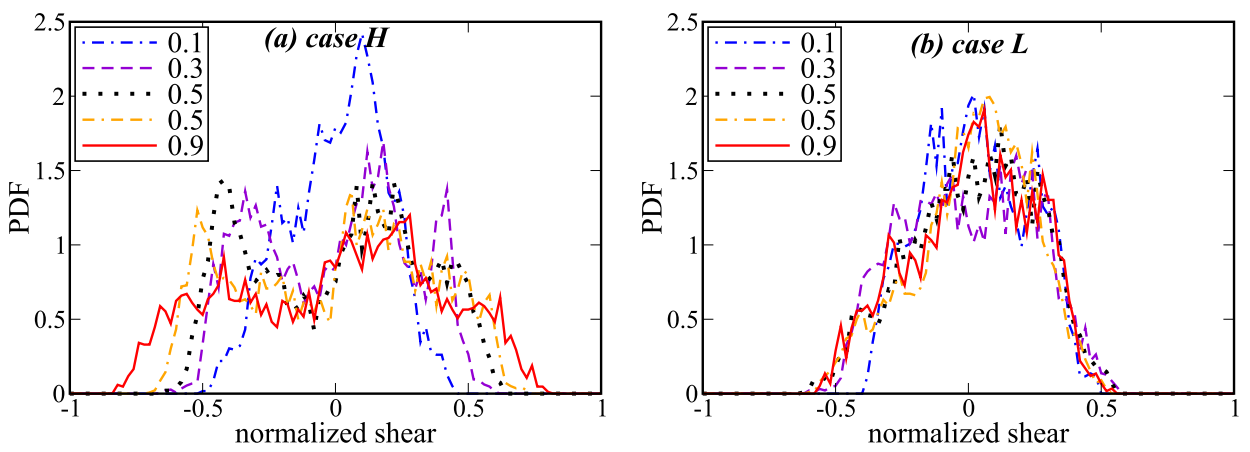

FIG. 4. Probability density functions for the normalized local shear $|\nabla \cdot \mathbf{u}|_{L}^{-1}(\partial u / \partial y)$ calculated in the middle of flamelets $(0.495<c(\mathbf{x}$, $t)<0.505)$ at various values of the Reynolds-averaged combustion progress variable $\bar{c}$, specified in legends, in cases (a) $\mathrm{H}$ and (b) L. 

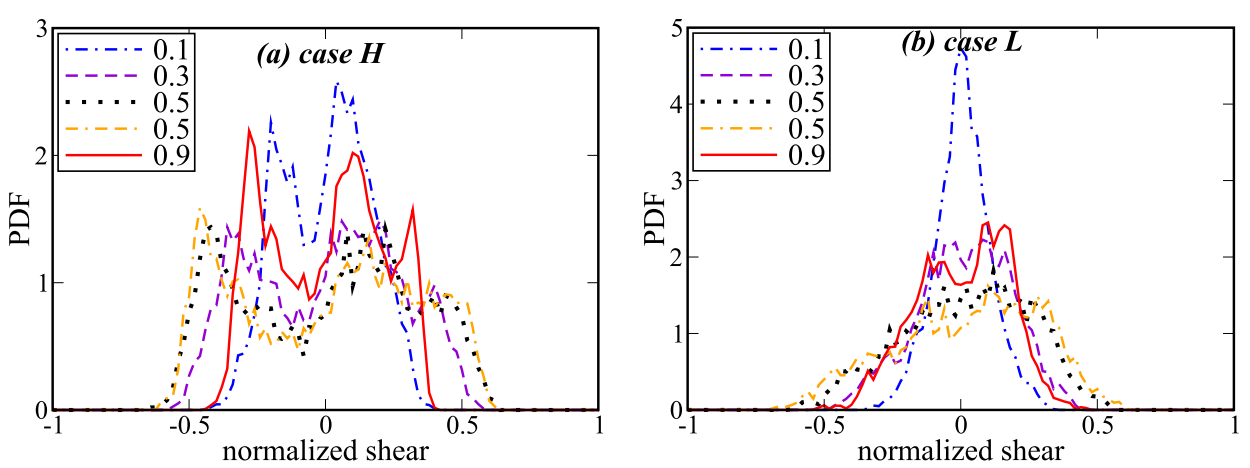

FIG. 5. Probability density functions for the normalized local shear $|\nabla \cdot \mathbf{u}|_{L}^{-1}(\partial u / \partial y)$ calculated in the middle of flame brushes, i.e., at $\bar{c}(x) \approx 0.5$, at various local $c(\mathbf{x}, t)$ specified in legends in cases (a) $\mathrm{H}$ and (b) L. the same factor of about four. Therefore, not only turbulence but also the combustion-induced thermal expansion affect the considered PDF's.

Figure 6 shows PDF's for the local shear associated with the magnitude $\left|\nabla_{t} u_{n}\right|$ of the tangential (to the local flamelet surface) gradient of the normal velocity, evaluated within flamelets at various $\bar{c}$ and normalized using $|\nabla \cdot \mathbf{u}|_{L}$. While the PDF's peak at $\left|\nabla_{t} u_{n}\right| /|\nabla \cdot \mathbf{u}|_{L} \ll 1$, they are sufficiently wide and there are non-negligible probabilities of finding the tangential gradient $\left|\nabla_{t} u_{n}\right|$ comparable with the normal gradient $|\nabla \cdot \mathbf{u}|_{L}$ in the ULFF. In case $\mathrm{H}$, the effect is much more pronounced at $\bar{c}=0.9$ due to appearance of unburned mixture fingers discussed elsewhere. ${ }^{32,38}$ Moreover, the PDF half-widths are smaller by a factor of about two in case $\mathrm{H}$ (with the exception of $\bar{c}=0.9$ ) when compared to case $\mathrm{L}$, whereas $|\nabla \cdot \mathbf{u}|_{L}$ is larger by a factor of about four in the former case. If the PDF's were solely controlled by turbulence, the H-half-widths would be smaller by approximately the same factor of about four. Therefore, Fig. 6 also implies that large magnitudes of the local shear within flamelets are substantially affected by the local thermal expansion.
The discussed effects are more pronounced for the local shear associated with the magnitude $\left|\mathbf{n} \cdot \nabla u_{t}\right|$ of the normal gradient of the tangential velocity. Indeed, Fig. 7(a) shows that its PDF's peak at non-zero values of $\left|\mathbf{n} \cdot \nabla u_{t}\right| / /\left.\nabla \cdot \mathbf{u}\right|_{L}$, which are increased from about 0.05 to 0.31 when $\bar{c}$ is increased from 0.1 to 0.9 . Comparison of Figs. 7(a) and 7(b) clearly indicates the influence of the density ratio and, hence, of combustioninduced thermal expansion on the PDF shape. At the same time, the fact that the PDF shapes are significantly different at $\sigma=7.53$ and 2.5, cf. Figs. 7(a) and 7(b), respectively, implies that the use of the laminar $|\nabla \cdot \mathbf{u}|_{L}$ to normalize $\left|\mathbf{n} \cdot \nabla u_{t}\right|$ is not sufficient to describe statistics of the latter gradient in the turbulent flames. In other words, it is difficult to model such statistics by directly using expressions valid for the laminar flame.

A physical mechanism of local shear generation due to combustion-induced thermal expansion is depicted in Figs. 8 and 9. The former figure shows iso-surfaces of (i) the peak rate $W\left(\mathbf{x}, t_{B}\right)$, i.e., the reaction surface, and (ii) $c\left(\mathbf{x}, t_{B}\right)$ $=0.01$ (flamelet leading edge) in planes $z=z_{B}$ and $y=y_{B}$ that cross an "extreme" point B that has been found using the following two constraints: (i) the extreme point B lies on a
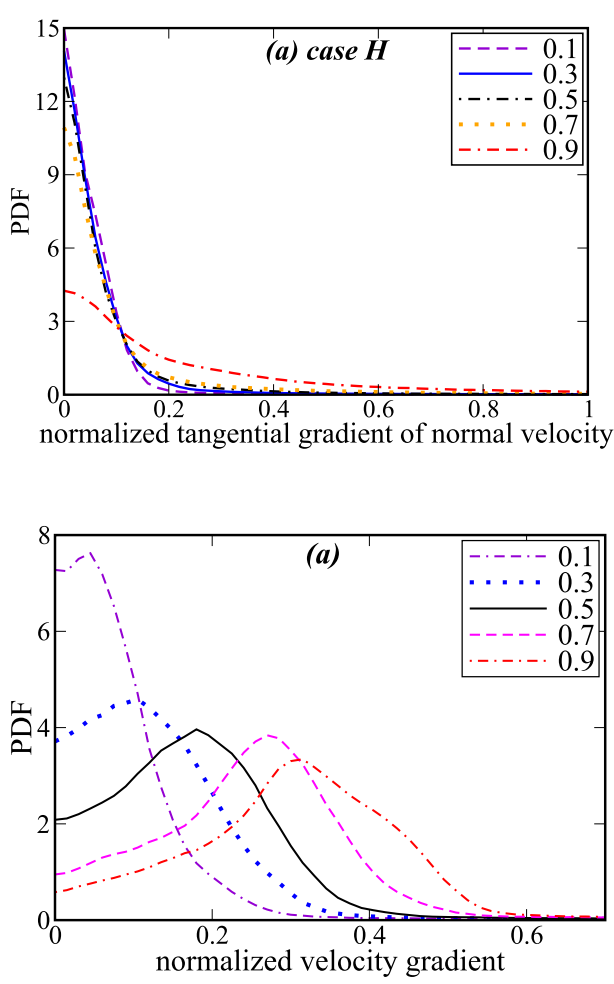
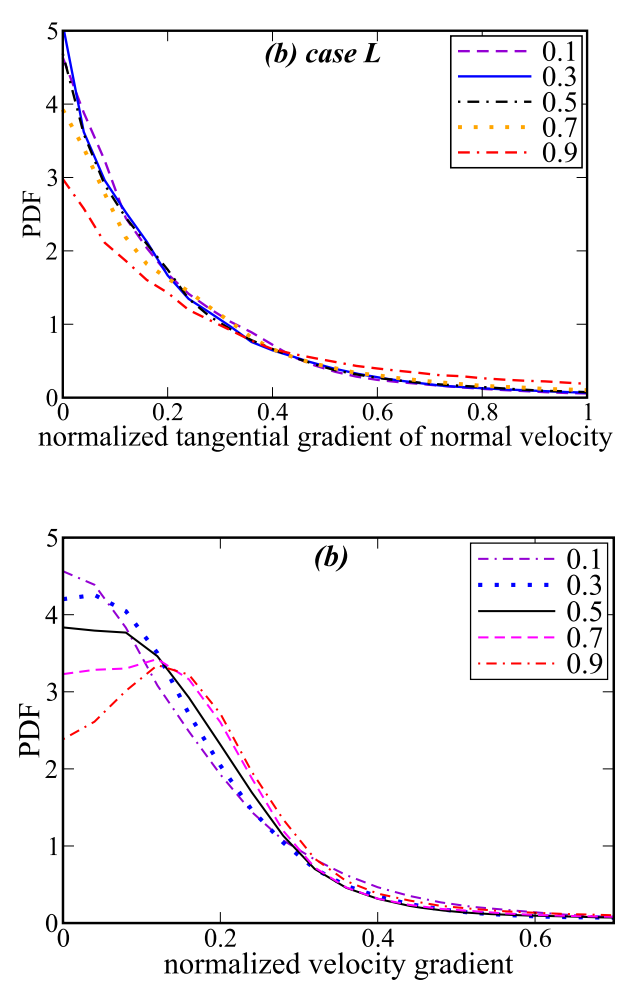

FIG. 6. Probability density functions for the magnitude $\left|\nabla_{t} u_{n}\right|$ of the tangential gradient of the normal velocity, evaluated within flamelets $(\epsilon<c(\mathbf{x}, t)<$ $1-\epsilon)$ at various values of the Reynoldsaveraged combustion progress variable $\bar{c}$, specified in legends, in cases (a) $\mathrm{H}$ and (b) $\mathrm{L}$. The gradient is normalized using $|\nabla \cdot \mathbf{u}|_{L}=(\sigma-1) S_{L} / \delta_{L}$.

FIG. 7. Probability density functions for the magnitude $\left|\mathbf{n} \cdot \nabla u_{t}\right|$ of the normal gradient of the tangential velocity, evaluated within flamelets $(\epsilon<c(\mathbf{x}, t)<1$ $-\epsilon)$ at various values of the Reynoldsaveraged combustion progress variable $\bar{c}$, specified in legends, in cases (a) $\mathrm{H}$ and (b) $\mathrm{L}$. The gradient is normalized using $|\nabla \cdot \mathbf{u}|_{L}=(\sigma-1) S_{L} / \delta_{L}$. 

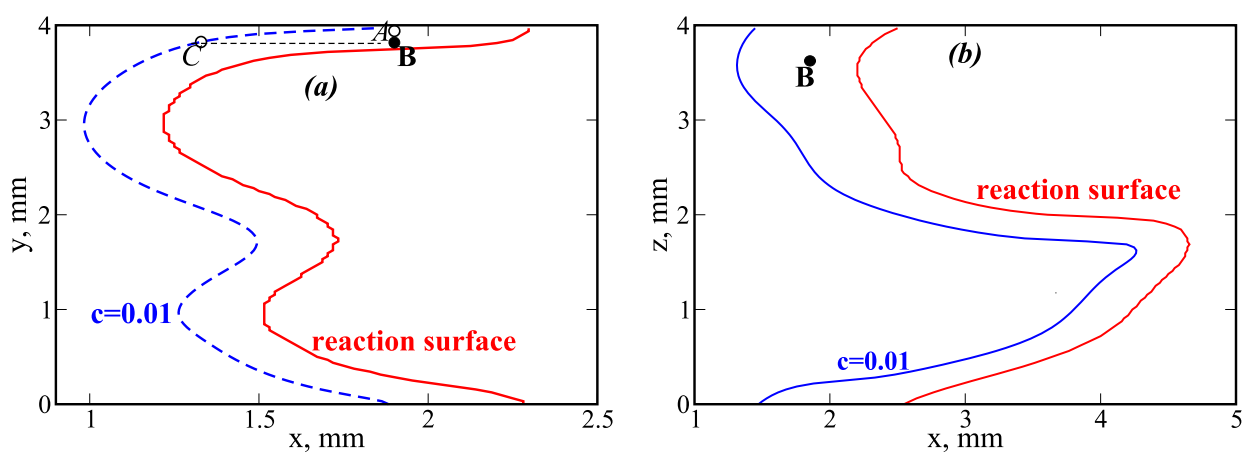

FIG. 8. Flame shapes in (a) $x y$ and (b) $x z$ planes that cross the extreme point $\mathrm{B}$ (black filled circle) characterized by $n_{y}$ $\approx 1$ and by a high magnitude $|\mathbf{n} \cdot \nabla u|$ of the normal (to the local flamelet) gradient of the axial velocity $u$, which is almost tangential to the flamelet in the vicinity of that point. Reaction surface $x$ $=x_{i}\left(y_{j}, z_{k}\right)$ is characterized by the maximal reaction rate $W(c)$ along a ray of $(y$ $=y_{j}, z=z_{k}$ ). Case $\mathrm{H}, \bar{c}=0.5$.

transverse plane $x=x_{B}$ that is most close to the mean flame surface $\bar{c}(x)=0.5$ and (ii) the extreme point $\mathrm{B}$ is characterized by the maximal (over various $y, z$, and $t$ ) magnitude $\left|\left\langle\mathbf{n} \cdot \nabla u \mid \sqrt{n_{y} n_{y}}>n^{*} \| \sqrt{n_{z} n_{z}}>n^{*}\right\rangle\right|$ of the normal (to the local flamelet) gradient of the axial velocity $u$ conditioned to either $\left|n_{y}\right|$ or $\left|n_{z}\right|$ being larger than a threshold value $n^{*}$. Since the threshold value is set to be very close to unity, i.e., $n^{*}=0.995$, the iso-surface $c\left(\mathbf{x}_{B}, t_{B}\right)=$ const. is almost parallel to the $x$-axis in the extreme point $\mathrm{B}$. In particular, the iso-surface is almost normal to the $y$-axis in Fig. 8(a).

Therefore, the local $x$ - or $z$-profiles of various quantities are almost tangential to the local flamelet, whereas the local $y$-profiles are almost normal to it. This feature of the extreme point B significantly facilitates exploring local flow perturbations because (i) coordinate transformations are not required to obtain the locally tangential or normal profiles and (ii) the perturbations are well pronounced due to selection of the maximal conditioned $|\mathbf{n} \cdot \nabla u|$. Accordingly, the solid, dashed, and dotted-dashed lines in Fig. 9 show instantaneous $x$ (tangential), $y$ (normal), and $z$ (tangential) profiles, respectively, i.e., $q(x, y$ $\left.=y_{B}, z=z_{B}, t=t_{B}\right), q\left(x=x_{B}, y, z=z_{B}, t=t_{B}\right)$, and $q\left(x=x_{B}, y=\right.$ $y_{B}, z, t=t_{B}$ ), respectively, of pressure $p$, see Fig. 9(a), velocity components $u$ (red curves), $v$ (black curves), $w$ (blue curves), see Fig. 9(b), and vorticity components $\omega_{x}$ (red curves), $\omega_{y}$ (black curves), $\omega_{z}$ (blue curves), see Fig. 9(c).

Figure 8(a) shows that the axial distance $\left|x_{B}-x_{C}\right|$ between point $\mathrm{B}$ and the flamelet leading edge, i.e., point $\mathrm{C}$, is sufficiently long; see the dashed straight line. On the contrary, a neighboring point $A$ characterized by the same axial coordinate, i.e., $x_{A}=x_{B}$, the same $z_{A}=z_{B}$, and by a lower value of the combustion progress variable, i.e., $c_{A}<c_{B}$, is close to the flamelet leading edge. Accordingly, an infinitesimal gas volume that comes to point B is likely to move within the flamelet during a time interval $\Delta t_{B}$, which is significantly longer than time interval $\Delta t_{A}$ required for another gas volume to move from the flamelet leading edge to point $\mathrm{A}$. When a fluid volume moves within the flamelet, the density in the volume is less than $\rho_{u}$ and decreases with increasing the local $c$. Consequently, the volume acceleration $\rho^{-1} \partial p / \partial x$ by the axial pressure gradient induced due to combustion in surrounding volumes, see the local axial profile $p\left(x, y=y_{B}, z=z_{B}, t=t_{B}\right)$ of pressure, shown as a red solid line in Fig. 9(a), is significantly stronger than axial acceleration that a volume of unburned reactants would experience under the influence of the same axial pressure gradient. Therefore, the infinitesimal volume associated with point $\mathrm{B}$ (A) is likely to be subject to an increased axial acceleration, see the local axial profile $u\left(x, y=y_{B}, z=z_{B}, t=t_{B}\right)$ of the axial velocity, shown as a red solid line in Fig. 9(b), during relatively long (short, respectively) time interval $\Delta t_{B}\left(\Delta t_{A}\right.$, respectively). As a result, $u_{B}$ is significantly larger than $u_{A}$, yielding a strong locally normal (i.e., in the $y$-direction) gradient of the axial velocity $u$, which is locally tangential to the reaction surface; see the locally normal profile of $u\left(x=x_{B}, y, z=z_{B}, t=t_{B}\right)$ shown as a red dashed line in Fig. 9(b). This physical mechanism appears to be responsible for the substantial widths and the locations of peaks of PDF's plotted in Fig. 7. It is basically similar to physical mechanisms suggested by Scurlock and Grover ${ }^{8}$ to explore generation of turbulence in combustion products due to local shear layers or by Libby and Bray ${ }^{40}$ to explain countergradient turbulent scalar transport in flames.

In addition to the axial pressure gradient and the gradient of the axial velocity, discussed above, there are significant locally normal (to the flamelet) gradients of (i) pressure, see the local profile of $p\left(x=x_{B}, y=y, z=z_{B}, t=t_{B}\right)$ shown as a black dashed line in Fig. 9(a) and (ii) the normal velocity $v$, see the local profile of $v\left(x=x_{B}, y=y, z=z_{B}, t=t_{B}\right)$ shown as
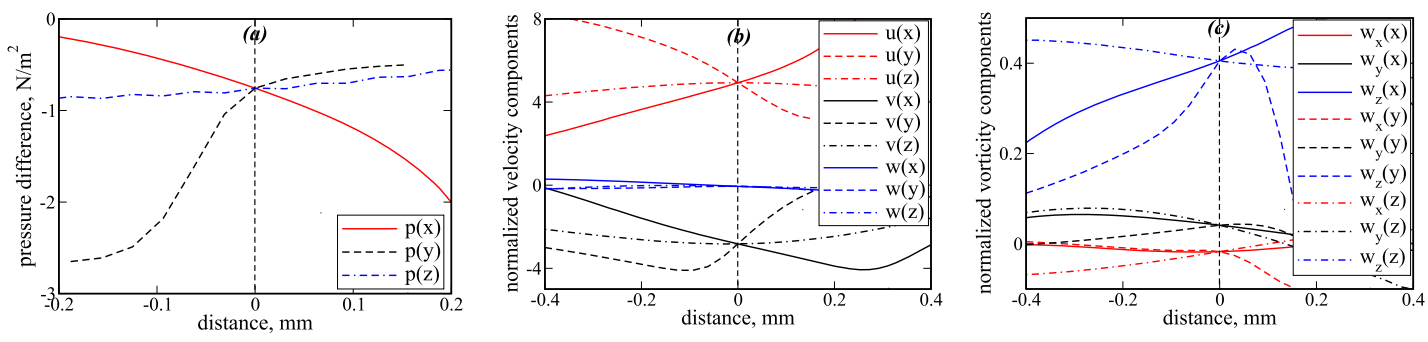

FIG. 9. Normalized profiles of (a) difference $\Delta p$ in the local pressure and pressure averaged over the inlet plane, and components of (b) velocity $\mathbf{u}=\{u, v, w\}$ and (c) vorticity $\omega=\left\{\omega_{x}, \omega_{y}, \omega_{z}\right\}$ vectors along the three axes. All profiles cross the extreme point B shown in Fig. 8, with distance being counted from this point. Negative distance is associated with lower (or higher) values of the combustion progress variable $c$ for the $x$ and $z$ (or $y$, respectively) profiles. The velocity and vorticity profiles are normalized using $S_{L}$ and $D_{u} / S_{L}$, respectively. 

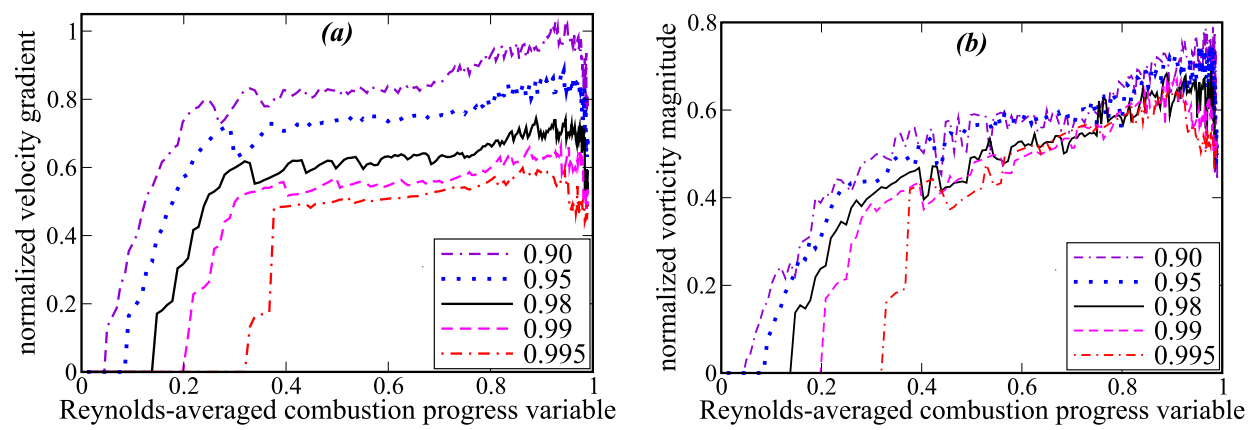

FIG. 10. (a) Maximal (over transverse plane and various instants) absolute values of the normal (to the local flamelet) gradient of the axial velocity $\left|\left\langle\mathbf{n} \cdot \nabla u \mid \sqrt{n_{y} n_{y}}>n^{*} \| \sqrt{n_{z} n_{z}}>n^{*}\right\rangle\right|$, normalized using $(\sigma-1) S_{L} / \delta_{L}$ and conditioned to either $\left|n_{y}\right|$ or $\left|n_{z}\right|$ being larger than a threshold $n^{*}$ specified in legends. (b) Magnitude $|\omega|$ of the vorticity vector, normalized using $D_{u} / S_{L}$ and evaluated in points where $\left|\left\langle\mathbf{n} \cdot \nabla u \mid \sqrt{n_{y} n_{y}}>n^{*} \| \sqrt{n_{z} n_{z}}>n^{*}\right\rangle\right|$ reaches the maximal values. Case $\mathrm{H}$.

a black dashed line in Fig. 9(b), in the vicinity of the extreme point B. The local gradients $\nabla_{y} p>0$ and $\nabla_{y} v>0$ result from the local density drop within the flamelet. However, the magnitude of the local change of the locally normal velocity $v$ in the locally normal direction $y$ is about $4 S_{L}$, see the black dashed line in Fig. 9(b), i.e., less than $\Delta u_{L}=6.5 S_{L}$, thus, indicating local perturbations in the velocity field when compared to the ULFF.

Moreover, the black solid line in Fig. 9(b) shows that the magnitude of the locally tangential gradient of the locally normal velocity, i.e., $|\partial v / \partial x|$, is also large in the extreme point $\mathrm{B}$, thus, explaining substantial widths of PDF's for $\nabla_{t} u_{n}$, plotted in Fig. 6. This observation is not surprising because the $z$-component $\omega_{z}=\partial v / \partial x-\partial u / \partial y$ of the vorticity vector $\omega$ is large in the vicinity of point $\mathrm{B}$; see the blue curves in Fig. 9(c). The point is that an angle between (i) vector $\nabla \rho$, which is almost parallel to the $y$-axis, as shown in Fig. 8(a), and (ii) vector $\nabla p$, whose components $\nabla_{x} p$ and $\nabla_{y} p$ have comparable magnitudes in point $\mathrm{B}$, cf. the red solid and black dashed lines in Fig. 9(a), is sufficiently large in this region. Consequently, vorticity is locally generated by a physical mechanism known as baroclinic torque and discussed in detail elsewhere. ${ }^{9,10,31,41-43}$

Figure 10 shows that significant perturbations (i.e., shear layers and regions with a high vorticity) of the local velocity field within flamelets that are almost normal to the mean flame brush exist at various $\bar{c}$ with the exception of the leading part $\left(\bar{c}<c^{*}\right)$ of the flame brush. An increase in the threshold normal $n^{*}$ results in increasing the boundary value $c^{*}$ because flamelets characterized by a very low $\left|n_{x}\right|$ do not reach regions characterized by a low $\bar{c}$.
However, such strong velocity perturbations induced due to thermal expansion are accompanied by relatively moderate perturbations of the flamelet scalar structure under conditions of the present simulations (adiabatic burning, $L e=1$, singlestep chemistry, and the reaction rate is solely controlled by $c$ ). While an increase in the distance between the reaction surface and flamelet leading edge is observed in the vicinity of the extreme point B in Fig. 8(b), Fig. 11 shows that the local perturbations of the normalized displacement speed

$$
\frac{\rho S_{d}}{\rho_{u} S_{L}}=\frac{\nabla \cdot(\rho D \nabla c)+W}{\rho_{u} S_{L}}
$$

and, especially, consumption velocity

$$
\frac{u_{c}}{\rho_{u} S_{L}}=\frac{\int_{-\delta}^{\delta} W d \zeta}{\rho_{u} S_{L}}
$$

are sufficiently weak in the vicinity of the extreme point $\mathrm{B}$, discussed earlier. Here, the distance $\delta$ is counted from the extreme point B in the $y$ direction. An increase in $u_{c} /\left(\rho_{u} S_{L}\right)$ from a value about unity in the vicinity of the extreme point $B$ to a value about 2.2 at distances larger than $0.4 \mathrm{~mm}$ is caused by the second reaction zone, which is shown in the bottom part of Fig. 8(a). The second jump in $u_{c} /\left(\rho_{u} S_{L}\right)$ is larger than unity due to inclination of the second reaction zone with respect to the $y$-axis along which integration in Eq. (8) is performed.

Figure 12 also indicates that local perturbations in both $|\nabla c|$ and $u_{c}$ are sufficiently weak in flamelet points characterized by a low $\left|n_{x}\right|$ and a strong local shear, i.e., a large $|\mathbf{n} \cdot \nabla u|$. The consumption velocity reported in Fig. 12(b) was evaluated using Eq. (8), with the distance $\delta$ being counted from the local
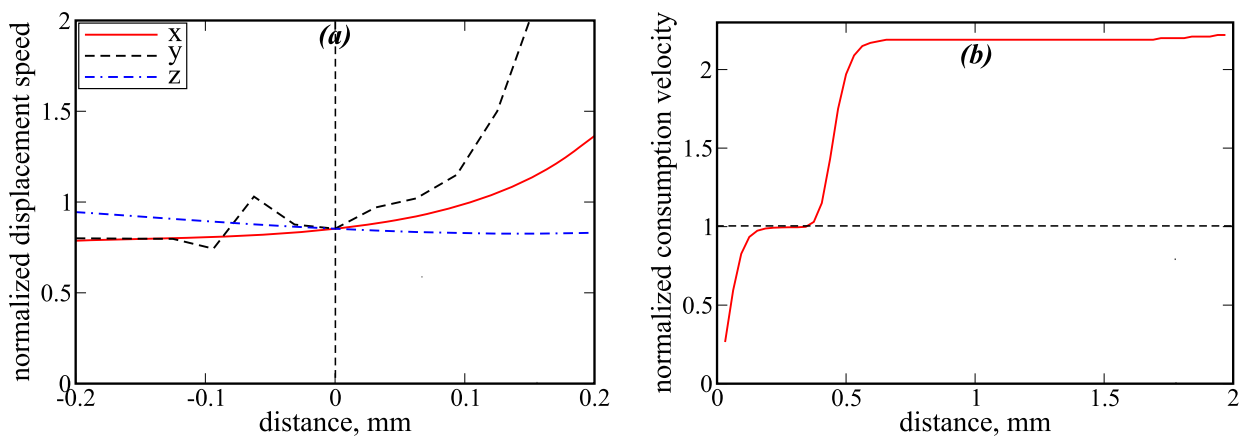

FIG. 11. Profiles of (a) the normalized local displacement speed $\rho S_{d} /\left(\rho_{u} S_{L}\right)$ vs. distance from the extreme point $\mathrm{B}$ in $x, y$, and $z$ directions and of (b) the normalized local consumption velocity $u_{c} /\left(\rho_{u} S_{L}\right)$ vs. distance in the $y$-direction. All profiles cross the extreme point $\mathrm{B}$ shown in Fig. 8. 


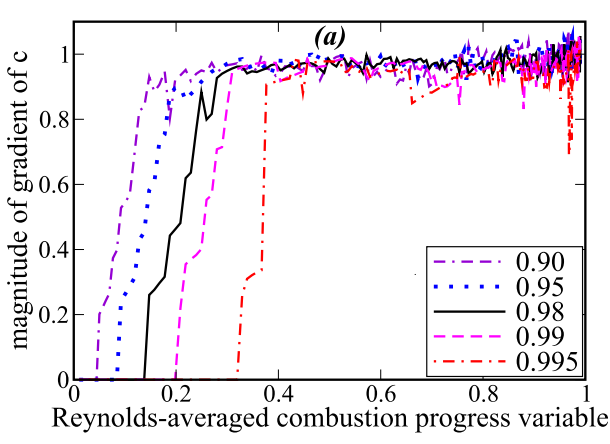

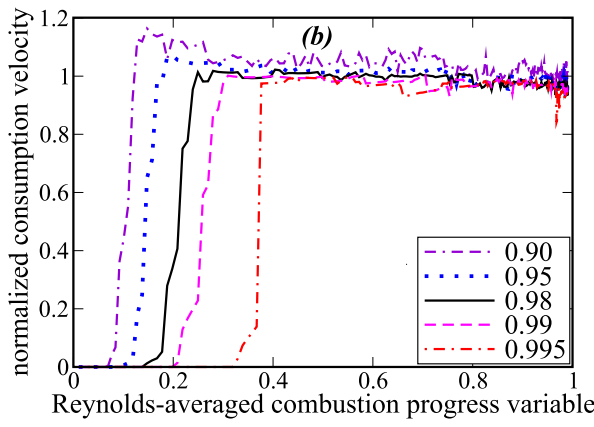

FIG. 12. (a) Local gradient $|\nabla c|$ of the combustion progress variable, normalized using $\delta_{L}$, and (b) local normalized consumption velocity $u_{c} /\left(\rho_{u} S_{L}\right)$ evaluated in points where $\mid\left\langle\mathbf{n} \cdot \nabla u \mid \sqrt{n_{y} n_{y}}\right\rangle$ $\left.n^{*} \| \sqrt{n_{z} n_{z}}>n^{*}\right\rangle \mid$ reaches the maximal (over various $y, z$, and $t$ ) values. Case $\mathrm{H}$, a threshold $n^{*}$ is specified in legends. peak of the reaction rate along either the $y$-axis if $\left|n_{y}\right|>\left|n_{z}\right|$ or the $z$-axis otherwise. Calculations of the local consumption velocity were terminated at a distance $\delta$ required in order for $u_{c}(\delta)$ to reach a plateau; see Fig. 11(b).

While neither Fig. 11 nor Fig. 12 indicates significant perturbations of the local scalar structure of flamelets in regions where the local velocity field is strongly perturbed, i.e., in flamelet zones characterized by a large normal gradient $\left|\left\langle\mathbf{n} \cdot \nabla u\left|\sqrt{n_{y} n_{y}}>n^{*}\right| \mid \sqrt{n_{z} n_{z}}>n^{*}\right\rangle\right|$ and a low $\left|n_{x}\right| \ll 1$, the lack of the former (scalar) perturbations may stem from too simple conditions of the present DNS. Under more realistic conditions that are more favorable for flamelet structure perturbations and even local quenching (e.g., $L e \neq 1$, heat losses, and/or complex combustion chemistry), strong perturbations of velocity and pressure fields could cause strong perturbations of the local flamelet structure, $S_{d}$, and $u_{c}$. This issue is worth further studying by analyzing appropriate DNS databases.

Finally, since the results reported in Figs. 8-12 were obtained by selecting extreme points characterized by the largest $\left|\left\langle\mathbf{n} \cdot \nabla u \mid \sqrt{n_{y} n_{y}}>n^{*} \| \sqrt{n_{z} n_{z}}>n^{*}\right\rangle\right|$ and $\left|n_{x}\right| \ll 1$, the statistical importance of the studied local effects may be put into question. To address such a concern, the following ratios

$$
R(g)=\frac{\iiint\left\langle\left. T|| \nabla_{t} u_{n}|=g| \nabla \cdot \mathbf{u}\right|_{L}\right\rangle d V_{f}}{\iiint T d V_{f}}
$$

were calculated for various values $g=\left|\nabla_{t} u_{n}\right| /|\nabla \cdot \mathbf{u}|_{L}$ of the normalized locally tangential gradient of the locally normal velocity, with the term $T$ being equal either to the magnitude
$\left|T_{\omega}\right|=\left|\rho^{-2} \nabla \rho \times \nabla p\right|$ of the baroclinic torque term in the transport equation for vorticity $\omega$ or to the baroclinic torque term $T_{\omega}=\rho^{-2} \omega \cdot(\nabla \rho \times \nabla p)$ in the transport equation for enstrophy $\boldsymbol{\omega} \cdot \boldsymbol{\omega}$. Integration in Eq. (9) was solely performed over flamelet volume $V_{f}$ where $\epsilon<c(\mathbf{x}, t)<1-\epsilon$. Accordingly, the two ratios $R_{\omega}$ and $R_{\omega}$ characterize relative contributions to vorticity and enstrophy, respectively, generation due to baroclinic torque from flamelet regions characterized by particular magnitudes $g-0.5 \Delta g \leq\left|\nabla_{t} u_{n}\right| /|\nabla \cdot \mathbf{u}|_{L}<g+0.5 \Delta g$ of the locally tangential (to the flamelet) gradient of the locally normal velocity $u_{n}$. Similar ratios were also evaluated for the magnitude $\left|\mathbf{n} \cdot \nabla u_{t}\right|$ of the locally normal gradient of the locally tangential velocity.

The dashed lines in Fig. 13(a) show that, while the ratio $R_{\omega}$ (the results obtained for $R_{\omega}$ are very similar and are not reported for brevity) has a sufficiently sharp peak at low $g=$ $\left|\nabla_{t} u_{n}\right| /|\nabla \cdot \mathbf{u}|_{L}$, notable values of $R_{\omega}$ are still observed at tangential gradients of the normal velocity, whose magnitudes $\left|\nabla_{t} u_{n}\right|$ are comparable with the magnitude $|\nabla \cdot \mathbf{u}|_{L}$ of the velocity gradient across the laminar flame. Therefore, contribution of flamelet regions associated with significant local shear $\left|\nabla_{t} u_{n}\right|$ to the total generation of vorticity due to baroclinic toque is not negligible. This trend is more pronounced in case $\mathrm{L}$, see the black dashed line, probably, because vorticity generation due to baroclinic torque is weak in other regions in this case characterized by a low density ratio.

Solid lines in Fig. 13(a) show that the ratio $R_{\omega}$ peaks at $g=\left|\mathbf{n} \cdot \nabla u_{t}\right| /|\nabla \cdot \mathbf{u}|$ about 0.3 in case $H$. Therefore, contribution of flamelet regions associated with significant local shear $\left|\mathbf{n} \cdot \nabla u_{t}\right|$ to the total generation of vorticity due to baroclinic toque is substantial in this case.
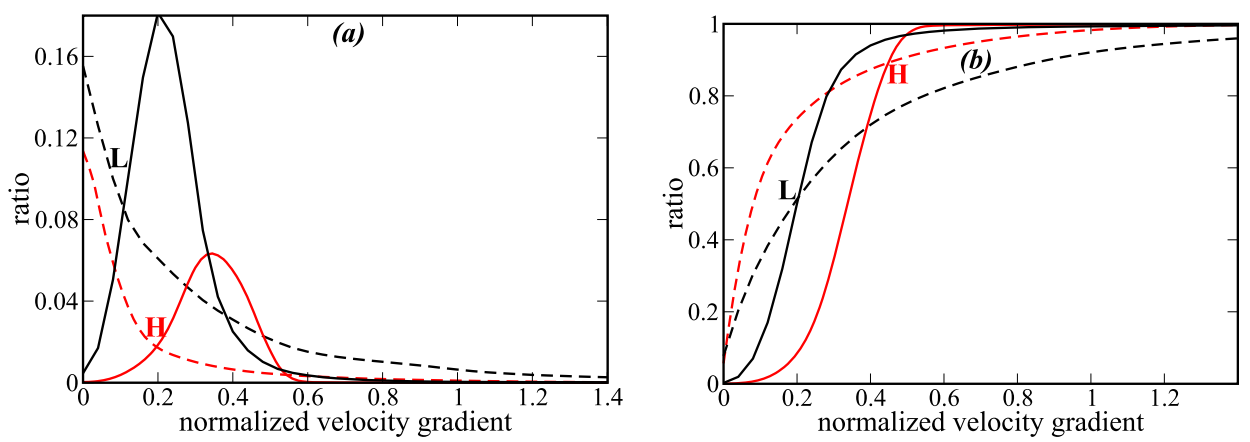

FIG. 13. Relative contributions to enstrophy generation due to baroclinic torque from flamelet regions characterized by (a) $g-0.5 \Delta g \leq\left|\nabla_{t} u_{n}\right| /|\nabla \cdot \mathbf{u}|_{L}<g+$ $0.5 \Delta g$ (dashed lines) or $g-0.5 \Delta g \leq \ln \cdot \nabla u_{t}|/ / \nabla \cdot \mathbf{u}|_{L}<g+0.5 \Delta g$ (solid lines) and (b) $\left|\nabla_{t} u_{n}\right| / /\left.\nabla \cdot \mathbf{u}\right|_{L}<g+0.5 \Delta g$ (dashed lines) or $\left|\mathbf{n} \cdot \nabla u_{t}\right| / /\left.\nabla \cdot \mathbf{u}\right|_{L}<g+0.5 \Delta g$ (solid lines) vs. the value $g$ of the locally tangential gradient of the locally normal velocity (dashed lines) or the locally normal gradient of the locally tangential velocity (solid lines), normalized with $|\nabla \cdot \mathbf{u}|_{L}$. 
Contribution of the strong-shear-regions to vorticity generation in the studied premixed turbulent flames is even more pronounced if ratios like

$$
\mathcal{R}(g)=\frac{\iiint\left\langle\left. T|| \nabla_{t} u_{n}|\leq g| \nabla \cdot \mathbf{u}\right|_{L}\right\rangle d V_{f}}{\iiint T d V_{f}},
$$

which is simply equal to the sum of the ratios $R$ for all tangential gradients weaker than $g|\nabla \cdot \mathbf{u}|_{L}$, are considered. Indeed, the dashed lines in Fig. 13(b) show that flamelet regions characterized by $\left|\nabla_{t} u_{n}\right|>0.2|\nabla \cdot \mathbf{u}|_{L}$ are responsible for about $20 \%$ of volume-integrated baroclinic torque term $T_{\omega}$ in case $\mathrm{H}$ and even $50 \%$ in case L. Moreover, the red solid line indicates that more than $50 \%$ of volume-integrated baroclinic torque term $T_{\omega}$ stem from flamelet regions characterized by $\left|\mathbf{n} \cdot \nabla u_{t}\right|$ $>0.3|\nabla \cdot \mathbf{u}|_{L}$ in case $\mathrm{H}$. Thus, flamelet regions characterized by a strong local shear $\left|\mathbf{n} \cdot \nabla u_{t}\right|$ induced due to thermal expansion may play a substantial role in vorticity generation in premixed turbulent flames.

\section{CONCLUSIONS}

DNS data obtained from turbulent premixed flames associated with the flamelet combustion regime were analyzed in order to explore differences between velocity and pressure variations (i) in flamelets in a weakly turbulent flow and (ii) in the counterpart laminar flame. Obtained results show that, while the scalar structure of flamelets is weakly perturbed when compared to the laminar flame under conditions of the present DNS, the local velocity, vorticity, and pressure fields within the flamelets may be significantly different from the local velocity, vorticity, and pressure fields, respectively, within the laminar flame. In particular, in the former case, there appear local shear layers characterized by large velocity gradients (both the tangential gradient of the normal velocity with respect to the flamelet surface and the normal gradient of the tangential velocity), whose magnitudes are comparable with the magnitude of the velocity gradient across the laminar flame. In flamelet zones characterized by a relatively large magnitude of the locally normal gradient of the tangential velocity, the local vorticity magnitude is also large and such zones contribute substantially to the overall (in the entire flame brush) generation of vorticity due to baroclinic torque. These results cast doubts on the validity of a simple common approach that consists in directly invoking expressions derived for the laminar flames in order to model the influence of combustion-induced thermal expansion on turbulent velocity and pressure fields.

It is worth stressing that such doubts concern solely velocity and pressure fields, whereas expressions derived for the thermo-chemical characteristics of laminar flames appear to be valid in the flamelet combustion regime addressed in the present paper. Indeed, Figs. 11 and 12 indicate weak perturbations in the local flamelet structure. Accordingly, the latter (i.e., thermo-chemical) expressions are widely used in exploring turbulent combustion; e.g., see recent models ${ }^{44,45}$ that invoke theoretical expressions for the cutoff length scale of the hydrodynamic instability ${ }^{46}$ of laminar premixed flames. Moreover, the doubts concern expressions derived by studying the unperturbed laminar premixed flames. Theoretical research into velocity and pressure fields within laminar premixed flames that propagate in more complicated flows could be of great interest for developing better models of the influence of combustion-induced thermal expansion on turbulence. The present paper calls for more attention to this problem.

As a future task, it will be of interest to explore whether or not the aforementioned shear layers can significantly affect the local flamelet structure in the cases of non-unity Lewis number, heat losses, and complex combustion chemistry. Moreover, investigation of the shear layers in more intense turbulence will also be of interest. It is worth remembering that the conditions of the present DNS $\left(u^{\prime} \ll \sigma S_{L}\right.$ in flame $\left.\mathrm{H}\right)$ are beneficial for effects due to combustion-induced thermal expansion, but such effects could be of less importance in the opposite case of $u^{\prime} \gg \sigma S_{L}$, as discussed elsewhere. ${ }^{9,10}$

\section{ACKNOWLEDGMENTS}

A.N.L. gratefully acknowledges the financial support by the Chalmers Area of Advance Transport and by the Combustion Engine Research Center (CERC). V.A.S. gratefully acknowledges the financial support by ONERA and by the Grant of the Ministry of Education and Science of the Russian Federation (Contract No. 14.G39.31.0001 of 13.02.2017).

${ }^{1}$ G. Damköhler, "Der einfuss der turbulenz auf die flammengeschwindigkeit in gasgemischen," Z. Elektrochem. 46, 601 (1940) [NACA TM 1112 (1947) (in English)].

${ }^{2}$ K. I. Schelkin, "On combustion in a turbulent flow," Zh. Tekh. Fiz. 13, 520 (1943) [NACA TM 1110 (1947) (in English)].

${ }^{3}$ S. B. Pope, "Small scales, many species and the manifold challenges of turbulent combustion," Proc. Combust. Inst. 34, 1 (2013).

${ }^{4}$ Turbulent Combustion Modeling, edited by T. Echekki and E. Mastorakos (Springer-Verlag, Berlin, Germany, 2011).

${ }^{5}$ Turbulent Premixed Flames, edited by N. Swaminathan and K. N. C. Bray (Cambridge University Press, Cambridge, UK, 2011).

${ }^{6}$ A. Lipatnikov, Fundamentals of Premixed Turbulent Combustion (CRC Press, Boca Raton, Florida, 2012).

${ }^{7}$ B. Karlovitz, D. W. Denniston, and F. E. Wells, "Investigation of turbulent flames," J. Chem. Phys. 19, 541 (1951).

${ }^{8}$ A. C. Scurlock and J. H. Grover, "Propagation of turbulent flames," Symp. (Int.) Combust. 4, 645 (1953).

${ }^{9}$ A. N. Lipatnikov and J. Chomiak, "Effects of premixed flames on turbulence and turbulent scalar transport," Prog. Energy Combust. Sci. 36, 1 (2010).

${ }^{10}$ V. A. Sabelnikov and A. N. Lipatnikov, "Recent advances in understanding of thermal expansion effects in premixed turbulent flames," Annu. Rev. Fluid Mech. 49, 91 (2017).

${ }^{11}$ L. Tian and R. P. Lindstedt, "The impact of dilatation, scrambling, and pressure transport in turbulent premixed flames," Combust. Theory Modell. 21, 1114 (2018).

${ }^{12}$ K. N. C. Bray, "Turbulent flows with premixed reactants," in Turbulent Reacting Flows, edited by P. A. Libby and F. A. Williams (Springer-Verlag, Berlin, Germany, 1980), pp. 115-183.

${ }^{13}$ R. Borghi, "Turbulent combustion modeling," Prog. Energy Combust. Sci. 14, 245 (1988).

${ }^{14}$ N. Peters, Turbulent Combustion (Cambridge University Press, Cambridge, UK, 2000).

${ }^{15}$ S. Nishiki, T. Hasegawa, R. Borghi, and R. Himeno, "Modeling of flamegenerated turbulence based on direct numerical simulation databases," Proc. Combust. Inst. 29, 2017 (2002).

${ }^{16} \mathrm{~S}$. Nishiki, T. Hasegawa, R. Borghi, and R. Himeno, "Modelling of turbulent scalar flux in turbulent premixed flames based on DNS databases," Combust. Theory Modell. 10, 39 (2006).

${ }^{17}$ H. Carlsson, R. Yu, and X.-S. Bai, "Flame structure analysis for categorization of lean premixed $\mathrm{CH}_{4}$ /air and $\mathrm{H}_{2}$ /air flames at high Karlovitz numbers: Direct numerical simulation studies," Proc. Combust. Inst. 35, 1425 (2015). 
${ }^{18}$ H. A. Uranakara, S. Chaudhuri, H. L. Dave, P. G. Arias, and H. G. Im, "A flame particle tracking analysis of turbulence-chemistry interaction in hydrogen-air premixed flames," Combust. Flame 163, 220 (2016).

${ }^{19}$ A. J. Aspden, M. J. Day, and J. B. Bell, "Three-dimensional direct numerical simulation of turbulent lean premixed methane combustion with detailed kinetics," Combust. Flame 166, 266 (2016).

${ }^{20}$ B. Bobbitt, S. Lapointe, and G. Blanquart, "Vorticity transformation in high Karlovitz number premixed flames,” Phys. Fluids 28, 015101 (2016).

${ }^{21}$ H. Wang, E. R. Hawkes, B. Zhou, J. H. Chen, Z. Li, and M. Aldén, "A comparison between direct numerical simulation and experiment of the turbulent burning velocity-related statistics in a turbulent methane-air premixed jet flame at high Karlovitz number," Proc. Combust. Inst. 36, 2045 (2017).

${ }^{22}$ S. Chaudhuri, H. Kolla, H. L. Dave, E. R. Hawkes, J. H. Chen, and C. K. Law, "Flame thickness and conditional scalar dissipation rate in a premixed temporal turbulent reacting jet," Combust. Flame 184, 273 (2017).

${ }^{23}$ Y. Minamoto, B. Yenerdag, and M. Tanahashi, "Morphology and structure of hydrogen-air turbulent premixed flames," Combust. Flame 192, 369 (2018).

${ }^{24}$ A. N. Lipatnikov, S. Nishiki, and T. Hasegawa, "DNS assessment of relation between mean reaction and scalar dissipation rates in the flamelet regime of premixed turbulent combustion," Combust. Theory Modell. 19, 309 (2015).

${ }^{25}$ Y. H. Im, K. Y. Huh, S. Nishiki, and T. Hasegawa, "Zone conditional assessment of flame-generated turbulence with DNS database of a turbulent premixed flame," Combust. Flame 137, 478 (2004).

${ }^{26}$ A. Mura, K. Tsuboi, and T. Hasegawa, "Modelling of the correlation between velocity and reactive scalar gradients in turbulent premixed flames based on DNS data," Combust. Theory Modell. 12, 671 (2008).

${ }^{27}$ A. Mura, V. Robin, M. Champion, and T. Hasegawa, "Small scale features of velocity and scalar fields in turbulent premixed flames," Flow Turbul. Combust. 82, 339 (2009).

${ }^{28}$ V. Robin, A. Mura, M. Champion, and T. Hasegawa, "Direct and indirect thermal expansion effects in turbulent premixed flames," Combust. Sci. Technol. 182, 449 (2010).

${ }^{29}$ V. Robin, A. Mura, and M. Champion, "Modeling of the effects of thermal expansion on scalar turbulent fluxes in turbulent premixed flames," J. Fluid Mech. 689, 149 (2011).

${ }^{30}$ K. N. C. Bray, M. Champion, P. A. Libby, and N. Swaminathan, "Scalar dissipation and mean reaction rates in premixed turbulent combustion," Combust. Flame 158, 2017 (2011).

${ }^{31}$ A. N. Lipatnikov, S. Nishiki, and T. Hasegawa, "A direct numerical simulation study of vorticity transformation in weakly turbulent premixed flames," Phys. Fluids 26, 105104 (2014).
${ }^{32}$ A. N. Lipatnikov, J. Chomiak, V. A. Sabelnikov, S. Nishiki, and T. Hasegawa, "Unburned mixture fingers in premixed turbulent flames," Proc. Combust. Inst. 35, 1401 (2015).

${ }^{33}$ A. N. Lipatnikov, V. A. Sabelnikov, S. Nishiki, T. Hasegawa, and N. Chakraborty, "DNS assessment of a simple model for evaluating velocity conditioned to unburned gas in premixed turbulent flames," Flow Turbul. Combust. 94, 513 (2015).

${ }^{34}$ V. A. Sabelnikov, A. N. Lipatnikov, N. Chakraborty, S. Nishiki, and T. Hasegawa, "A transport equation for reaction rate in turbulent flows," Phys. Fluids 28, 081701 (2016).

${ }^{35}$ V. A. Sabelnikov, A. N. Lipatnikov, N. Chakraborty, S. Nishiki, and T. Hasegawa, "A balance equation for the mean rate of product creation in premixed turbulent flames," Proc. Combust. Inst. 36, 1893 (2017).

${ }^{36}$ A. N. Lipatnikov, V. A. Sabelnikov, S. Nishiki, and T. Hasegawa, "Flamelet perturbations and flame surface density transport in weakly turbulent premixed combustion," Combust. Theory Modell. 21, 205 (2017).

${ }^{37}$ A. N. Lipatnikov, V. A. Sabelnikov, N. Chakraborty, S. Nishiki, and T. Hasegawa, "A DNS study of closure relations for convection flux term in transport equation for mean reaction rate in turbulent flow," Flow Turbul. Combust. 100, 75 (2018).

${ }^{38}$ A. N. Lipatnikov, J. Chomiak, V. A. Sabelnikov, S. Nishiki, and T. Hasegawa, "A DNS study of the physical mechanisms associated with density ratio influence on turbulent burning velocity in premixed flames," Combust. Theory Modell. 22, 131 (2018).

${ }^{39}$ R. H. Kraichnan, "Decay of isotropic turbulence in the direct-interaction approximation," Phys. Fluids 7, 1030 (1964).

${ }^{40}$ P. A. Libby and K. N. C. Bray, "Countergradient diffusion in premixed turbulent flames," AIAA J. 19, 205 (1981).

${ }^{41}$ P. E. Hamlington, A. Y. Poludnenko, and E. S. Oran, "Interactions between turbulence and flames in premixed reacting flows," Phys. Fluids 23, 125111 (2011).

${ }^{42}$ P. E. Hamlington, A. Y. Poludnenko, and E. S. Oran, "Intermittency in premixed turbulent reacting flows," Phys. Fluids 24, 075111 (2012).

${ }^{43}$ N. Chakraborty, I. Konstantinou, and A. N. Lipatnikov, "Effects of Lewis number on vorticity and enstrophy transport in turbulent premixed flames," Phys. Fluids 28, 015109 (2016).

${ }^{44}$ S. Chaudhuri, V. Akkerman, and C. K. Law, "Spectral formulation of turbulent flame speed with consideration of hydrodynamic instability," Phys. Rev. E 84, 026322 (2011).

${ }^{45}$ S. Chaudhuri, V. Akkerman, and C. K. Law, "Accelerative propagation and explosion triggering by expanding turbulent premixed flames," Phys. Rev. E 87, 023008 (2013).

${ }^{46}$ L. D. Landau and E. M. Lifshitz, Fluid Mechanics (Pergamon Press, Oxford, UK, 1987). 